\title{
Spin relaxation of radicals in cryptochrome and its role in avian magnetoreception
}

Cite as: J. Chem. Phys. 145, 035104 (2016); https://doi.org/10.1063/1.4958624

Submitted: 16 May 2016 . Accepted: 16 June 2016 . Published Online: 20 July 2016

Susannah Worster (D), Daniel R. Kattnig, and P. J. Hore (D)

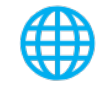

\section{ARTICLES YOU MAY BE INTERESTED IN}

Floquet theory of radical pairs in radiofrequency magnetic fields

The Journal of Chemical Physics 145, 124117 (2016); https://doi.org/10.1063/1.4963793

An improved semiclassical theory of radical pair recombination reactions

The Journal of Chemical Physics 139, 124106 (2013); https://doi.org/10.1063/1.4821817

Sub-millitesla magnetic field effects on the recombination reaction of flavin and ascorbic acid radicals

The Journal of Chemical Physics 145, 085101 (2016); https://doi.org/10.1063/1.4961266 


\title{
Spin relaxation of radicals in cryptochrome and its role in avian magnetoreception
}

\author{
Susannah Worster, Daniel R. Kattnig, and P. J. Hore ${ }^{\text {a) }}$ \\ Department of Chemistry, Physical and Theoretical Chemistry Laboratory, University of Oxford, \\ South Parks Road, Oxford OX1 3QZ, United Kingdom
}

(Received 16 May 2016; accepted 16 June 2016; published online 20 July 2016)

Long-lived spin coherence and rotationally ordered radical pairs have previously been identified as key requirements for the radical pair mechanism of the avian magnetic compass sense. Both criteria are hard to meet in a biological environment, where thermal motion of the radicals creates dynamic disorder and drives efficient spin relaxation. This has long been cited as a major stumbling block of the radical pair hypothesis. Here we combine Redfield relaxation theory with analytical solutions to a rotational diffusion equation to assess the impact of restricted rotational motion of the radicals on the operation of the compass. The effects of such motions are first investigated generally in small, model systems and are then critically examined in the magnetically sensitive flavin-tryptophan radical pair that is formed photochemically in the proposed magnetoreceptor protein, cryptochrome. We conclude that relaxation is slowest when rotational motion of the radicals within the protein is fast and highly constrained; that in a regime of slow relaxation, the motional averaging of hyperfine interactions has the potential to improve the sensitivity of the compass; and that consideration of motional effects can significantly alter the design criteria for an optimal compass. In addition, we demonstrate that motion of the flavin radical is likely to be compatible with its role as a component of a functioning radical-pair compass, whereas the motion of the tryptophan radical is less ideal, unless it is particularly fast. Published by AIP Publishing. [http://dx.doi.org/10.1063/1.4958624]

\section{INTRODUCTION}

In addition to visual and olfactory cues, ${ }^{1-3}$ migratory birds navigate using a magnetic compass with which they are able to detect the direction of the Earth's magnetic field. ${ }^{4,5}$ There is a substantial body of experimental and theoretical evidence ${ }^{6-10}$ to support the proposal that the compass sense is mediated by a magnetically sensitive chemical reaction. ${ }^{11}$ The idea is that a photo-chemically generated radical pair undergoes coherent singlet-triplet interconversion driven by hyperfine interactions between the electron and nuclear spins in each radical. ${ }^{12} \mathrm{~A}$ weak external magnetic field modifies the interconversion frequencies, with the extent of the change depending on the strength of the field and its orientation relative to the radical pair. Information about the direction of the external field is recorded in the concentration of a signalling state molecule produced by spin-selective reactions of the singlet and triplet states. This mechanism is consistent with several properties of the avian compass, for example, that it requires blue-green light to function ${ }^{13,14}$ and detects only the inclination and not the polarity of the Earth's field. ${ }^{4}$ Disruption of the compass by electromagnetic noise has also been cited as diagnostic evidence for the radical pair mechanism. ${ }^{8,15,16}$

In 2000, cryptochrome was suggested as a candidate magnetoreceptor. ${ }^{17}$ Under blue-light irradiation, cryptochrome undergoes fast intra-protein electron transfer to form a radical pair composed of the semiquinone form of the

\footnotetext{
a) Author to whom correspondence should be addressed. Electronic mail: peter.hore@chem.ox.ac.uk.
}

flavin adenine dinucleotide cofactor $\left(\mathrm{FAD}^{\bullet-}\right)$ and an oxidized tryptophan residue $\left(\mathrm{TrpH}^{\bullet+}\right) .{ }^{18}$ Cryptochromes are widespread across phyla and have been found to play a role in many blue-light-dependent signalling pathways, including circadian photoreception in insects. ${ }^{19}$ Crucially for its proposed role as a magnetoreceptor, cryptochrome has been found in photoreceptor cells in the retinas of birds, ${ }^{20-22}$ where it co-localises with neuronal activity markers during night-time magnetic orientation. ${ }^{23}$ Spectroscopic analysis $^{18,24-26}$ and theoretical simulations ${ }^{27-30}$ have shown that cryptochrome exhibits many of the properties necessary to function as a magnetoreceptor.

For a radical pair reaction to function as a compass, it must meet a number of structural and kinetic requirements (reviewed in Refs. 30 and 12). We focus in this work on two in particular: first, that the radical pair must, to some extent, have a fixed orientation within the retina and second, that spin-coherence must persist long enough to allow detection of an anisotropic response to the external field. Both conditions are crucial for a functional compass but have the capacity to be adversely affected by molecular motion, making them potentially difficult to realise in a biological environment.

The key principle of the radical pair mechanism is that the outcome of the reaction depends on the direction of the external field relative to the radical pair. Maintaining a degree of molecular alignment is the obvious way to translate this outcome into meaningful information about the bird's orientation. ${ }^{17,31,32}$ The radical pair must have a fixed alignment within the cell, which in turn must be aligned within the retina. Previous studies have shown that a relatively large 
degree of static disorder $\left(30^{\circ}-40^{\circ}\right.$ mean angular deviation) could be introduced within ensembles of radical pairs without losing more than half the compass sensitivity. ${ }^{33,34}$ Others have argued that populations of partially aligned radical pairs could be generated by selectively exciting molecules that are correctly aligned with the electric field vector of the incoming light. ${ }^{35}$ Both arguments deal exclusively with detecting the average singlet yield produced by an ensemble of radical pairs, each of which has a different, fixed orientation relative to the external field. However, little has been done so far to establish the impact of dynamic disorder, where every individual radical pair samples a range of different orientations during its lifetime.

In addition to creating dynamic disorder, motion of the magnetoreceptor molecule has severe implications for the spin-coherence lifetime of the radical pair. To accumulate the maximum anisotropy in the reaction yield, the coherence needs to persist for about $1 \mu$ s (the reciprocal of the Larmor frequency of a free electron in an Earth-strength magnetic field). ${ }^{30}$ Furthermore, the rate of spin relaxation should be similar to or slower than the rate at which the radical pair reacts or recombines to the ground state. If the coherence lifetime is significantly shorter than the reaction lifetime, most of the reaction product will be formed from radical pairs that exist in the directionally independent equilibrium ratio of $25 \%$ singlet, $75 \%$ triplet. This dilutes the directional information provided by those radical pairs that reacted whilst still far from equilibrium, with a field-dependent probability of being in the singlet or triplet state. Unfortunately, modulation of the magnetic interactions within the radical pair system by molecular motion is an unavoidable source of spin relaxation.

The question of how a sufficiently long coherence time could be achieved in the warm, noisy environment of a cell has, therefore, long been an obstacle for the radical pair magnetoreception hypothesis. ${ }^{31}$ Using Lindblad operators to include relaxation, several theoretical studies have shown that although dephasing processes are generally detrimental, they can be tolerated to some extent and under certain conditions can even be beneficial. ${ }^{36,37}$ However, such phenomenological studies provide no information about the physical conditions that would be required to create a minimally damaging or beneficial dephasing regime. In 2010, Lau et al. reported a study of the degree of relaxation induced by rotational tumbling of a protein containing a radical pair with a single nuclear spin. ${ }^{38}$ It concluded that reasonable compass sensitivity could be preserved if the stochastic rotations of the magnetoreceptor protein were of small to moderate amplitude and had a correlation time greater than about a quarter of the radical pair lifetime. Here we extend the investigation further to focus exclusively on the wobbling motions of the individual radicals within the magnetoreceptor protein. The details of these motions and the spin relaxation they generate have recently been explored for a plant cryptochrome using all-atom molecular dynamics simulations. ${ }^{39}$ Internal motions, only involving small sections of the protein, were shown to have shorter correlation times than the whole-molecule rotations considered by Lau et al., making it appropriate to use Redfield theory to create a compact description of the resulting spin relaxation. ${ }^{40-42}$ By combining Redfield theory with analytical solutions to the rotational diffusion equation, we are able to treat restricted motion of larger spin systems than have previously been considered. We examine the degree of spin relaxation that could be induced by the internal rotations of radicals within a magnetoreceptor protein and determine the sorts of motion that are most beneficial or least detrimental to its function as a magnetoreceptor.

The present work is further motivated by the recent discovery that many long-lived radical pairs are capable of producing sharp "spikes" in the reaction yield. ${ }^{43}$ These features could provide a previously missing explanation for the astonishing precision of the avian compass; birds are able to detect the axis of the Earth's field to within $5^{\circ}$ or less. ${ }^{44,45}$ For a spike to exist, the coherence lifetime would need to be at least $10 \mu \mathrm{s}$ - an order of magnitude longer than previously thought. As such, the spikes have the potential to be particularly susceptible to spin relaxation. We examine here the degree of rotational diffusion that spike-producing radicals can withstand and discuss the results in the context of a flavin-tryptophan magnetoreceptor.

\section{METHODS}

\section{A. Spin dynamics}

We model a radical pair generated in a pure singlet state, after which the system oscillates between its singlet and triplet states under the influence of Zeeman and hyperfine interactions. During this time, relaxation processes act to return the spin system to its equilibrium state. From the singlet state, the radical pair is able to recombine to the ground state, whilst the triplet state reacts spin-selectively to form a distinct product. We assume throughout that these two reactions occur with identical first-order rate constants. Although this is unlikely to be true in a real system, it greatly simplifies the calculations and does not obscure the behaviour we wish to study. ${ }^{43}$ The performance of the compass does not depend strongly on the ratio of the two rate constants providing they are not too dissimilar. ${ }^{43}$ Molecular motion is included by allowing one radical to undergo restricted rotational diffusion within an ordering potential while the other remains stationary.

The evolution of the radical pair spin system, described by the density matrix $\hat{\rho}(t)$, is modelled by a stochastic Liouville equation, ${ }^{46}$

$$
\begin{aligned}
\frac{\mathrm{d} \hat{\rho}(t)}{\mathrm{d} t} & =-\left(\mathrm{i} \hat{H}_{0}+k \hat{\hat{E}}-\hat{\hat{R}}\right) \hat{\rho}(t)-\hat{\hat{R}} \hat{\rho}_{\mathrm{eq}}(t) \\
& =-\hat{\hat{L}} \hat{\rho}(t)-\hat{\hat{R}} \hat{\mathrm{eq}}_{\mathrm{eq}}(t),
\end{aligned}
$$

where $\hat{\hat{H}}_{0}$ is the commutation superoperator that describes the coherent spin evolution of the system under the influence of Zeeman and hyperfine interactions, $k$ is the rate constant for the reactions of the singlet and triplet states, $\hat{\hat{E}}$ is the identity superoperator, and $\hat{\hat{R}}$ is a Redfield relaxation superoperator, ${ }^{40-42}$ which incorporates all the information about the relaxation behaviour. The interactions contained in $\hat{\hat{H}}_{0}$ are very small compared to the thermal energy $k_{\mathrm{B}} T$, so that to a very good approximation the equilibrium density matrix $\hat{\rho}_{\text {eq }}(t)$ (which is time-dependent due to the recombination 
reactions) is proportional to the identity matrix. Consequently, $\hat{\hat{R}} \hat{\rho}_{\text {eq }} \approx 0$ and this term will not be considered further.

Relaxation in this system can occur via many different pathways and hence cannot be defined simply by means of spin-lattice $\left(T_{1}\right)$ and spin-spin $\left(T_{2}\right)$ relaxation times. ${ }^{47}$ Instead the relaxation behaviour is characterised by its impact on the fractional yield of radical pairs recombining from the singlet state (the "singlet yield") for different external field directions.

The singlet yield can be calculated as ${ }^{48}$

$$
\Phi_{\mathrm{S}}=k \int_{0}^{\infty} p_{\mathrm{S}}(t) \mathrm{d} t=k\left\langle\hat{P}^{\mathrm{S}}\left|\hat{\hat{L}}^{-1}\right| \hat{\rho}(0)\right\rangle
$$

where $p_{\mathrm{S}}(t)=\operatorname{Tr}\left(\hat{\rho}(t) \hat{P}^{\mathrm{S}}\right)$ is the time-dependent singlet probability, $\hat{P}^{\mathrm{S}}$ is the singlet projection operator, and $\hat{\rho}(0)$ is the initial density matrix. For a spin system with $M$ nuclear spin states, $\hat{\rho}(0)=\hat{P}^{\mathrm{S}} / M$.

In Redfield theory, a perturbative master equation is derived for the spin system, under the assumption of weak system-environment coupling, retaining terms up to second order in the stochastically fluctuating part of the spin Hamiltonian. ${ }^{40-42}$ The approach requires that we first identify the unperturbed, time-independent component of the Hamiltonian,

$$
\begin{aligned}
\hat{H}_{0}= & -\gamma_{\mathrm{e}} B_{0}\left[\left(\hat{S}_{1 z}+\hat{S}_{2 z}\right) \cos \theta+\left(\hat{S}_{1 x}+\hat{S}_{2 x}\right) \sin \theta \cos \varphi+\left(\hat{S}_{1 y}+\hat{S}_{2 y}\right) \sin \theta \sin \varphi\right] \\
& +\sum_{\substack{i=1,2 \\
j=1 \ldots N}} a_{0, j}^{(0)} \hat{\mathbf{S}}_{i} \cdot \hat{\mathbf{I}}_{i, j}+\sum_{\substack{j \in \text { static } \\
\text { radical }}}\left(\sum_{m=-2}^{2} \hat{T}_{m, j}^{(2)} \phi_{m, j}\right)+\sum_{\substack{j \in \text { moving } \\
\text { radical }}}\left(\sum_{m=-2}^{2} \hat{T}_{0, j}^{(2)} \phi_{m, j}\left\langle\mathfrak{D}_{0, m}^{(2)}(t)\right\rangle_{\text {av }}\right) .
\end{aligned}
$$

$\hat{\mathbf{I}}_{i, j}$ and $\hat{\mathbf{S}}_{i}$ are, respectively, the spin operators for the $j$ th nucleus in the radical pair and the unpaired electron $i=1,2$ to which it is coupled. The first term is the Zeeman interaction between the unpaired electrons and the Earth's field, where $B_{0}$ is the field strength (taken to be $50 \mu \mathrm{T}$ ), $\gamma_{\mathrm{e}}$ is the gyromagnetic ratio, and $\theta$ and $\varphi$ are the polar and azimuthal angles that relate the magnetic field vector to the molecular axes (Fig. 1(a)). The Zeeman interaction is treated as isotropic, which is an excellent approximation for organic radicals (which normally have small $g$-tensor anisotropy) provided the lifetime of the spin coherence is less than $100 \mu$ s.

The second, third, and fourth terms in Eq. (3) are, respectively, the isotropic hyperfine interactions between the electron and the nuclei in each radical, the anisotropic hyperfine interactions in the static radical, and the average anisotropic hyperfine interactions in the moving radical. ${ }^{49}$ For simplicity, we assume that the radicals are sufficiently far apart that dipolar and exchange interactions between the two electrons are negligible. $\hat{T}_{m, j}^{(2)}$ are irreducible spherical tensor operators and $\mathfrak{D}_{m, m^{\prime}}^{(2)}(t)=\mathfrak{D}_{m, m^{\prime}}^{(2)}(\Omega)=\mathfrak{D}_{m, m^{\prime}}^{(2)}(\Omega(t))$ are elements of the second rank Wigner matrices, as functions of the three Euler angles, $\Omega(t)=(\alpha(t), \beta(t), \gamma(t))$, describing the orientation of the moving radical in the laboratory frame at time $t$ (Fig. 1(a)). We define the laboratory frame by the molecular axis system of the moving radical in its lowest energy orientation. Within each radical, the hyperfine interactions are positioned using the functions ${ }^{50}$

$$
\phi_{m, j}=\sum_{n=-2,0,2} a_{n, j}^{(2)} \mathfrak{D}_{m, n}^{(2)}\left(\Omega_{\mathrm{pos}}\right),
$$

where the Euler angles $\Omega_{\text {pos }}$ describe the rotation from the principal axis frame of the hyperfine tensor to the molecular frame (Fig. 1(b)). A method for extracting the angles $\Omega_{\text {pos }}$ from the hyperfine tensors is described in the supplementary material $\mathrm{S} 1 .^{51}$ The form of each hyperfine interaction is described by three parameters (Eqs. (5)-(7)),

$$
\begin{gathered}
\text { Isotropic component: } a_{0}^{(0)}=\frac{A_{x x}+A_{y y}+A_{z z}}{3}, \\
\text { Axiality: } a_{0}^{(2)}=\frac{2 A_{z z}-\left(A_{x x}+A_{y y}\right)}{\sqrt{6}}, \\
\text { Rhombicity: } a_{ \pm 2}^{(2)}=\frac{A_{x x}-A_{y y}}{2},
\end{gathered}
$$

where $A_{q q}$ are the eigenvalues of the hyperfine tensor, with $A_{z z} \geq A_{y y} \geq A_{x x}$. Each hyperfine tensor can also be divided into an isotropic component, $a_{\text {iso }}=a_{0}^{(0)}($ Eq. (5)) and an anisotropic part with eigenvalues $\left[T_{x x}, T_{y y}, T_{z z}\right]$. In its diagonal form, the full hyperfine tensor has principal components $\left[A_{x x}, A_{y y}, A_{z z}\right]=\left[a_{\text {iso }}+T_{x x}, a_{\text {iso }}+T_{y y}, a_{\text {iso }}+T_{z z}\right]$. The isotropic part of the tensor is unaffected by molecular rotation, whereas the magnitude of each component of the anisotropic part is always reduced by partial cancellation between different orientations of the radical.

As the moving radical wobbles within the ordering potential, the Euler angles defining its orientation in the laboratory frame change, altering the strength and direction of the field experienced by the electron due to the magnetic nuclei in the same radical. These small fluctuations perturb the static system and are described by the following time-dependent spin Hamiltonian:

$\hat{H}_{1}=\sum_{j \in \text { moving }}\left[\sum_{m=-2}^{2} \sum_{m^{\prime}=-2}^{2} \hat{T}_{m^{\prime}, j}^{(2)} \phi_{m, j}\left(\mathfrak{D}_{m^{\prime}, m}^{(2)}(t)-\left\langle\mathfrak{D}_{m^{\prime}, m}^{(2)}(t)\right\rangle_{\mathrm{av}}\right)\right]$.

Using Redfield theory, the evolution of the density matrix under this perturbation is given by the following set of coupled 
(A)

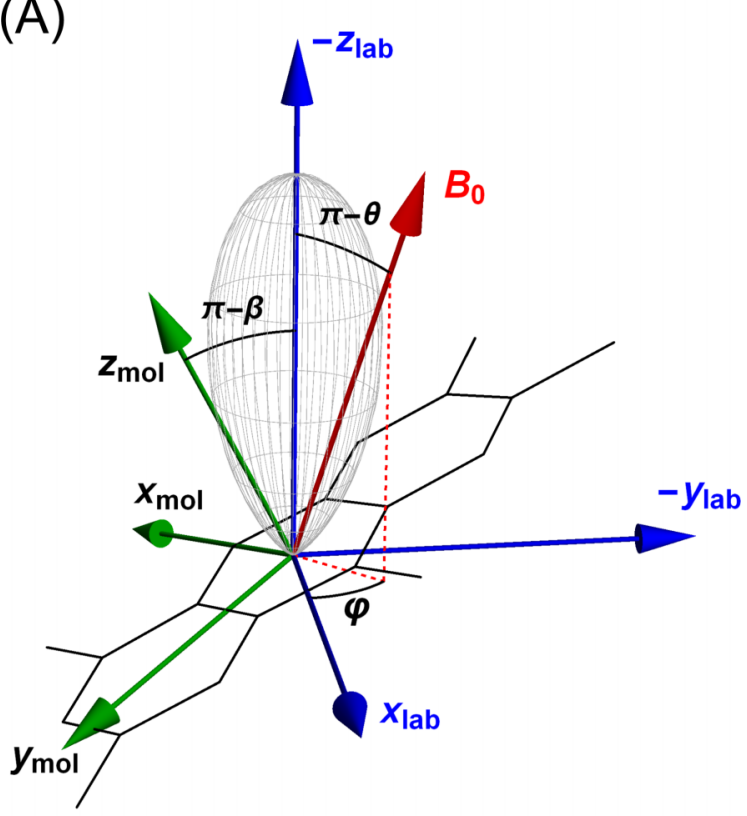

(B)

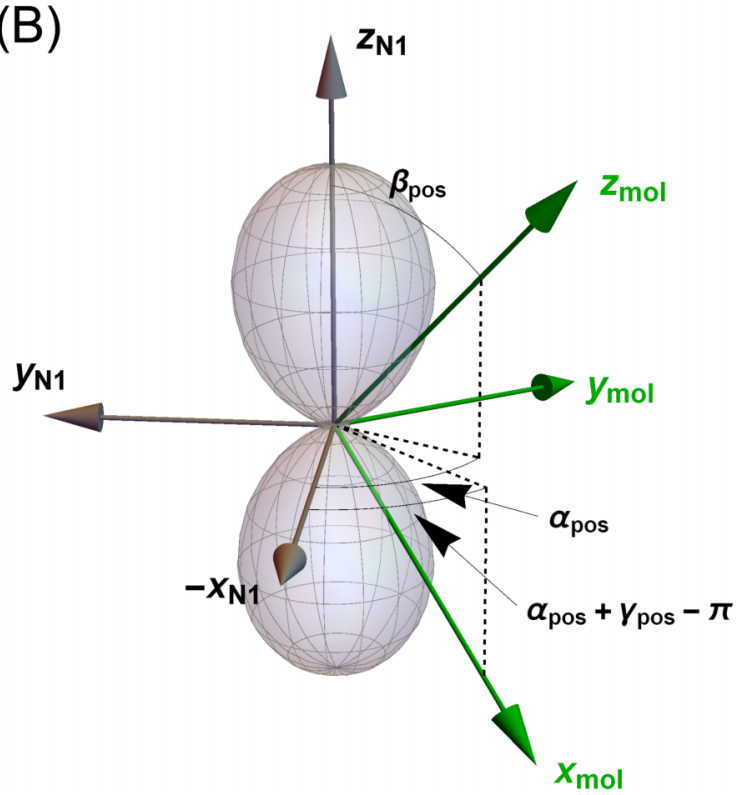

FIG. 1. Representation of the rotational diffusion of the flavin radical within an ordering potential, $U(\beta)$, of the type defined in Eq. (15), with $A=15$. $U(\beta)$ is represented here by the grey surface; the distance from the origin to the surface is proportional to the Boltzmann weight $\exp (-U(\beta))$ for the molecular $z$ axis being oriented in that direction. The position of the molecular axes (green) in the laboratory frame (blue) is specified by the Euler angles $\Omega(t)=(\alpha(t), \beta(t), \gamma(t))$. The orientation of magnetic field (red) is given by the angles $\theta$ and $\varphi$. (b) Orientation of the N1 hyperfine tensor in the molecular frame. The rotation from the principal axis frame (grey) to the molecular frame (green) is defined by the Euler angles $\Omega_{\text {pos }}(t)=\left(\alpha_{\text {pos }}(t), \beta_{\text {pos }}(t), \gamma_{\text {pos }}(t)\right)$. differential equations: $:^{40,50}$

$$
\frac{\mathrm{d} \rho_{\alpha \alpha^{\prime}}(t)}{\mathrm{d} t}=-\mathrm{i}\left[\hat{H}_{0}, \hat{\rho}(t)\right]_{\alpha \alpha^{\prime}}-k \rho_{\alpha \alpha^{\prime}}(t)+\sum_{\beta \beta^{\prime}} R_{\alpha \alpha^{\prime} \beta \beta^{\prime}} \rho_{\beta \beta^{\prime}}(t),
$$

where $\left[\hat{H}_{0}, \hat{\rho}(t)\right]_{\alpha \alpha^{\prime}}=\omega_{\alpha \alpha^{\prime}} \rho_{\alpha \alpha^{\prime}}(t)$ and

$$
\begin{aligned}
R_{\alpha \alpha^{\prime} \beta \beta^{\prime}}= & -\sum_{i, j \in \operatorname{moving}} \sum_{n, m}\left[\sum_{\gamma} J_{n m}^{i j}\left(\omega_{\gamma \beta}\right) \hat{T}_{m, i}^{(2), \alpha \gamma} \hat{T}_{n, j}^{(2) \dagger, \gamma \beta} \delta_{\alpha^{\prime} \beta^{\prime}}\right. \\
& -J_{n m}^{i j}\left(\omega_{\left.\beta^{\prime} \alpha^{\prime}\right)}\right) \hat{T}_{m, i}^{(2), \alpha \beta} \hat{T}_{n, j}^{(2) \dagger, \beta^{\prime} \alpha^{\prime}} \\
& -J_{n m}^{i j}\left(\omega_{\alpha \beta}\right) \hat{T}_{n, j}^{(2) \dagger, \alpha \beta} \hat{T}_{m, i}^{(2), \beta^{\prime} \alpha^{\prime}} \\
& \left.+\sum_{\gamma} J_{n m}^{i j}\left(\omega_{\beta^{\prime} \gamma}\right) \hat{T}_{n, j}^{(2) \dagger, \beta^{\prime} \gamma} \hat{T}_{m, i}^{(2), \gamma \alpha^{\prime}} \delta_{\alpha \beta}\right] .
\end{aligned}
$$

$J_{n m}^{i j}(\omega)$ are spectral densities, discussed in detail in Sec. II B, and $\omega_{\alpha \beta}$ is the energy gap between the $|\alpha\rangle$ and $|\beta\rangle$ eigenstates of $\hat{H}_{0} . \hat{T}_{m, i}^{(2), \alpha \beta} \equiv\left\langle\alpha\left|\hat{T}_{m, i}^{(2)}\right| \beta\right\rangle$ are matrix elements of the irreducible spherical tensor operators, in the eigenbasis of $\hat{H}_{0}$.

In its derivation, Redfield theory makes the key assumption of Markovian behaviour, which limits its validity to cases where the correlation time for the motion is much shorter than the time scale of coherent evolution of the spin system. We were able to confirm the validity of the Redfield approach for correlation times up to $10 \mathrm{~ns}$ by comparing our results with the stochastic Liouville equation treatment used by Lau et al. ${ }^{38}$ (see supplementary material S2). ${ }^{51}$ This is sufficient for our purposes as we wish to investigate internal motions, which are likely to have shorter correlation times than the 20-30 ns predicted for rigid-body rotations of the whole magnetoreceptor protein. ${ }^{38}$

To aid the computation, the secular approximation was also made. This did not significantly impact the results of our calculation. An explanation and derivation of the approximation are given in the supplementary material S3. ${ }^{51}$

\section{B. Spectral densities}

The Redfield relaxation matrix is constructed from products of irreducible spherical tensor operators and spectral densities of the form

$$
J_{m n}^{i j}(\omega)=\sum_{n^{\prime} m^{\prime}} \int_{0}^{\infty} \phi_{m^{\prime}, i} \phi_{n^{\prime}, j}^{*} g_{m m^{\prime} n n^{\prime}}(t) \mathrm{e}^{\mathrm{i} \omega t} \mathrm{~d} t .
$$

$g_{m m^{\prime} n n^{\prime}}(t)$ are correlation functions characterising the average difference between the orientation of the radical at time 0 and a later time $t$. They are given by

$$
\begin{aligned}
g_{m m^{\prime} n n^{\prime}}(t) & =\left\langle\left[\mathfrak{D}_{m, m^{\prime}}^{(2)}\left(\Omega_{0}\right)-\left\langle\mathfrak{D}_{m, m^{\prime}}^{(2)}\left(\Omega_{0}\right)\right\rangle_{\mathrm{av}}\right]\left[\mathfrak{D}_{n, n^{\prime}}^{(2)}(\Omega)-\left\langle\mathcal{D}_{n, n^{\prime}}^{(2)}(\Omega)\right\rangle_{\mathrm{av}}\right]^{*}\right\rangle \\
& =\iint\left[\mathfrak{D}_{m, m^{\prime}}^{(2)}\left(\Omega_{0}\right)-\left\langle\mathcal{D}_{m, m^{\prime}}^{(2)}\left(\Omega_{0}\right)\right\rangle_{\mathrm{av}}\right]\left[\mathfrak{D}_{n, n^{\prime}}^{(2)}(\Omega)-\left\langle\mathcal{D}_{n, n^{\prime}}^{(2)}(\Omega)\right\rangle_{\mathrm{av}}\right]^{*} P\left(\Omega_{0}\right) P\left(\Omega_{0} \mid \Omega, t\right) \mathrm{d} \Omega_{0} \mathrm{~d} \Omega,
\end{aligned}
$$


where $P(\Omega)$ is the equilibrium probability that the radical has orientation $\Omega$, assuming a Boltzmann distribution of orientations within the ordering potential, $U(\Omega) . P\left(\Omega_{0} \mid \Omega, t\right)$ is the conditional probability that the radical has orientation $\Omega$ at time $t$ given that it had orientation $\Omega_{0}$ at time $t=0 .{ }^{52}$

For an asymmetric molecule rotating in a uniaxial liquid crystalline phase (a situation identical in its physics to the one considered here), the correlation functions may be analytically derived from the form of the ordering potential by solving the following differential equation for the conditional probability: ${ }^{52}$

$$
\frac{\partial P\left(\Omega_{0} \mid \Omega, t\right)}{\partial t}=-\boldsymbol{L} \boldsymbol{D}\left[\boldsymbol{L}+\boldsymbol{L} \frac{U(\Omega)}{k_{\mathrm{B}} T}\right] P\left(\Omega_{0} \mid \Omega, t\right) .
$$

The product of terms in front of $P\left(\Omega_{0} \mid \Omega, t\right)$ makes up the diffusion operator, where $\boldsymbol{L}=\left(L_{x}, L_{y}, L_{z}\right)$ is a dimensionless angular momentum operator and $\boldsymbol{D}=\operatorname{diag}\left(D_{x}, D_{y}, D_{z}\right)$ is the diffusion tensor

$$
\begin{aligned}
& \boldsymbol{D}=\varepsilon\left(\begin{array}{lll}
1 & 0 & 0 \\
0 & 1 & 0 \\
0 & 0 & \eta
\end{array}\right), \\
& \varepsilon=\frac{D_{x}+D_{y}}{2}, \quad \eta=\frac{2 D_{z}}{D_{x}+D_{y}} .
\end{aligned}
$$

$D_{x}=D_{y}=\varepsilon$ is the rate constant for the rotational diffusion ("wobbling") of the molecular $z$ axis; $D_{z}=\varepsilon \eta$ is the rate constant for diffusion around the $z$ axis. The ordering potential $U(\Omega)$ in Eq. (13) was chosen to have the uniaxial form

$$
\frac{U(\beta)}{k_{\mathrm{B}} T}=A \cos ^{2}\left(\frac{\beta}{2}\right),
$$

in which $A$ is a positive constant that determines the gradient of $U(\beta) . U(\beta)$ can also be expressed as a linear combination of normalized Wigner functions, $\left\{\mathfrak{D}_{q, p}^{(J)}\right\}$, whose behaviour under the angular momentum operators is well-defined

$$
\frac{U(\beta)}{k_{\mathrm{B}} T}=\frac{A}{2}\left(\mathfrak{D}_{0,0}^{(0)}(0, \beta, 0)+\mathfrak{D}_{0,0}^{(1)}(0, \beta, 0)\right) .
$$

Although motion over the barrier at $\beta=0$ is possible, we use values of $A$ large enough that the radical moves in what is effectively a simple harmonic potential, $U(\beta) / k_{\mathrm{B}} T \approx$ $A(\beta-\pi)^{2} / 4$, centred at $\beta=\pi$. Table I lists the values of $A$ for a series of potentials that permit an increasing range of molecular orientations.

Notice that although the wobbling of the $z$ axis is constrained, the radical undergoes free diffusion around its
TABLE I. Coefficients for potentials of different strengths. The mean angular deviation from the laboratory frame $z$ axis is calculated as $\langle\beta\rangle=\int_{0}^{\pi} \beta \sin (\beta) \exp \left(-U(\beta) / k_{\mathrm{B}} T\right) \mathrm{d} \beta / \int_{0}^{\pi} \sin (\beta) \exp \left(-U(\beta) / k_{\mathrm{B}} T\right) \mathrm{d} \beta$.

\begin{tabular}{lccccc}
\hline \hline$A$ & 400 & 100 & 50 & 27 & 15 \\
\hline$\langle\pi-\beta\rangle(\operatorname{deg})$ & 5.1 & 10.2 & 14.4 & 19.7 & 26.7 \\
$J_{\max }$ & 30 & 24 & 24 & 12 & 12 \\
\hline \hline
\end{tabular}

$z$ axis. Although not necessarily realistic, the latter motion has relatively little effect on a radical (e.g., the flavin radical studied below) whose hyperfine tensors are approximately symmetrical around the $z$ axis.

A symmetrized form of Eq. (13) is solved in matrix form in a basis of normalized Wigner functions $\left\{\mathfrak{D}_{q, p}^{(J)}\right\}$. The size of the basis set used limits the precision of the solution, with increasing numbers of higher rank Wigner functions being required to describe the diffusion for larger values of $A$. The maximum rank of Wigner function used to ensure convergence within each potential is given in Table I. For uniaxial potentials, of the type treated in this work, there is no coupling between basis functions with different $q$. The diffusion matrix can therefore be constructed and solved in independent blocks, labelled by $q$. The result is an analytical expression for the symmetrized conditional probability $\tilde{P}\left(\Omega_{0} \mid \Omega, t\right)=P^{-1 / 2}(\Omega) P\left(\Omega_{0} \mid \Omega, t\right) P^{1 / 2}\left(\Omega_{0}\right)$ as a linear combination of normalised Wigner functions, with coefficients that decay exponentially in time. The final expression for the correlation functions (see below) only contains the eigensolutions of blocks for which $|q|(|m|$ in Eq. (18)) is less than or equal to the maximum rank of the interactions in the Hamiltonian $(L=2)$. This limits the number of blocks of the diffusion matrix that need to be constructed.

The conditional probability satisfies the equilibrium condition

$$
\lim _{t \rightarrow \infty}\left[P\left(\Omega_{0} \mid \Omega, t\right)\right]=P(\Omega) .
$$

Assuming that the system starts from an equilibrium distribution of orientations within the ordering potential, an expression for $P\left(\Omega_{0}\right)$ can be found by taking the long-time limit of $P\left(\Omega_{0} \mid \Omega, t\right)$. Full expressions and derivations of all the probabilities are given by Tarroni and Zannoni. ${ }^{52}$ Substitution into Eq. (12) gives the final expression for the correlation functions as

$$
\begin{aligned}
& g_{m m^{\prime} n n^{\prime}}(t)=g_{m m^{\prime} n n^{\prime}}(t) \delta_{m n}=\sum_{J, J^{\prime}, J^{\prime \prime}, J^{\prime \prime \prime}, p, p^{\prime}, K} \frac{\sqrt{(2 J+1)\left(2 J^{\prime}+1\right)\left(2 J^{\prime \prime}+1\right)\left(2 J^{\prime \prime \prime}+1\right)}}{25} \\
& \times\left(X^{m}\right)_{J p, K}\left(X^{m}\right)_{J^{\prime} p^{\prime}, K}\left(X^{m}\right)_{J^{\prime \prime} m^{\prime}-p, K}\left(X^{m}\right)_{J^{\prime \prime \prime} n^{\prime}-p^{\prime}, K} \\
& \times C_{J^{\prime \prime \prime}, 0, J^{\prime}, m}^{2, m} C_{J^{\prime \prime \prime}, n^{\prime}-p^{\prime}, J^{\prime}, p^{\prime}}^{2, n^{\prime}} C_{J^{\prime \prime}, 0, J, m}^{2, m} C_{J^{\prime \prime}, m^{\prime}-p, J, p}^{2, m^{\prime}} \mathrm{e}^{t \varepsilon r_{K}^{m}} \\
& -\left\langle\mathfrak{D}_{m, m^{\prime}}^{(2)}(0)\right\rangle_{\mathrm{av}}\left\langle\mathfrak{D}_{m, n^{\prime}}^{(2)}(t)\right\rangle_{\mathrm{av}} \text {; } \\
& =\sum_{J, J^{\prime}, J^{\prime \prime}, J^{\prime \prime \prime}, p, p^{\prime}, K} \Gamma\left(K, m, m^{\prime}, n^{\prime}\right) \mathrm{e}^{t \varepsilon r_{K}^{m}}-\left\langle\mathfrak{D}_{m, m^{\prime}}^{(2)}(0)\right\rangle_{\mathrm{av}}\left\langle\mathfrak{D}_{m, n^{\prime}}^{(2)}(t)\right\rangle_{\mathrm{av}},
\end{aligned}
$$


where $\left(X^{m}\right)_{J p, K}$ is the element of the $K$ th eigenvector of the symmetrized diffusion operator matrix (see Eq. (13)) corresponding to the basis function $\mathfrak{D}_{m, p}^{(J)}$ and $r_{K}^{m}$ is the corresponding eigenvalue. $C_{J_{1}, m_{1}, J_{2}, m_{2}}^{J, m}$ are Clebsch-Gordan coefficients that evaluate standard integrals over sets of three Wigner functions. They are represented here using the convention adopted by Varshalovich et al. ${ }^{53}$

In Eq. (18), the correlation functions are expressed as sums of decaying exponentials (note that the eigenvalues $r_{K}^{m}$ are negative). In the limit $t \rightarrow \infty$, all terms tend to zero, except those with $r_{K}^{m}=0$. These terms correspond to the time-independent equilibrium value $\left\langle\mathfrak{D}_{m, m^{\prime}}^{(2)}(0)\right\rangle_{\mathrm{av}}\left\langle\mathfrak{D}_{m, n^{\prime}}^{(2)}(t)\right\rangle_{\mathrm{av}}$, which has deliberately been subtracted from the correlation functions (Eq. (12)), in line with the definition of the timedependent Hamiltonian (Eq. (8)).

For $r_{K}^{m}=0$, the first term of $g_{m m^{\prime} n n^{\prime}}$ can, by simple observation, be factorised into a product of two identically formed components, which are readily assigned to the two Wigner function averages, $\left\langle\mathfrak{D}_{m, m^{\prime}}^{(2)}(0)\right\rangle_{\text {av }}$ and $\left\langle\mathfrak{D}_{m, n^{\prime}}^{(2)}(t)\right\rangle_{\text {av }}$. The resulting formula for the time-averaged Wigner functions,

$$
\begin{aligned}
\left\langle\mathfrak{D}_{m, m^{\prime}}^{(2)}(t)\right\rangle_{\mathrm{av}}= & \sum_{J, J^{\prime}, p} \frac{\sqrt{(2 J+1)\left(2 J^{\prime}+1\right)}}{5}\left(\hat{X}^{(0)}\right)_{J p, 0} \\
& \times\left(\hat{X}^{(0)}\right)_{J^{\prime} p^{\prime}, 0} C_{J^{\prime}, 0, J, 0}^{2,0} C_{J^{\prime}, m^{\prime}-p, J^{\prime} p}^{2, m^{\prime}}
\end{aligned}
$$

is used to construct $\hat{H}_{0}$ (Eq. (3)). Note that $\left\langle\mathfrak{D}_{m, m^{\prime}}^{(2)}(\Omega)\right\rangle_{\text {av }}$ is zero unless $m=0$ and that this has been explicitly built into the last term in Eq. (3). As $\left\langle\mathcal{D}_{m, m^{\prime}}^{(2)}(\Omega)\right\rangle_{\text {av }}$ can also be evaluated analytically using

$$
\left\langle\mathfrak{D}_{m, m^{\prime}}^{(2)}(\Omega)\right\rangle_{\mathrm{av}}=\int P(\Omega) \mathfrak{D}_{m, m^{\prime}}^{(2)}(\Omega) \mathrm{d} \Omega,
$$

comparison of the values obtained from Eq. (19) or (20) was used as a means of confirming that a sufficiently large basis set had been used to form the diffusion operator matrix.

The imaginary part of the spectral density, as defined in Eq. (11), corresponds to a dynamic frequency shift that is very small and was therefore neglected in all calculations. The remaining, real part of the spectral density is given by ${ }^{54}$

$$
\operatorname{Re}\left(J_{m n}^{i j}(\omega)\right)=\sum_{K, m^{\prime}, n^{\prime}} \frac{-\varepsilon r_{K}^{m} \phi_{m^{\prime}, i} \phi_{n^{\prime}, j} \Gamma\left(K, m, m^{\prime}, n^{\prime}\right)}{\left(\varepsilon r_{K}^{m}\right)^{2}+\omega^{2}} \delta_{m n} \quad \text { with } r_{K}^{m} \neq 0 .
$$

The advantage of the method described above is that it allows us to calculate the effect of relaxation on the singlet yield based purely on physically meaningful parameters: the lifetime of the radical pair, the rate and extent of its motion, and the strength and direction of the external magnetic field.

\section{RESULTS}

\section{A. Small model systems}

Simulating the behaviour of realistic multi-nuclear spin systems is often expensive in terms of the computer time and memory requirements. ${ }^{55,56}$ Consequently, we explore the general effects of motion in small model systems before examining specific examples of more realistic radical pairs.

Following Hiscock et al. ${ }^{43}$ we start by considering a "toy" radical pair with a single spin-1 nucleus in each radical. The nucleus in one radical has a hyperfine coupling with principal components $[-0.2,-0.2,1.7659] \mathrm{mT}$. These values are loosely based on the hyperfine tensor of the N5 nitrogen in $\mathrm{FAD}^{\bullet-}$ and are well-designed for producing a spike in the singlet yield. Throughout this work, we refer to the radical principally responsible for producing the spike as the primary radical. The nucleus in the secondary radical in the toy model has an axially anisotropic hyperfine tensor, $[0,0,1.0812] \mathrm{mT}$, modelled on the indole nitrogen in $\mathrm{TrpH}^{\bullet+}$. In their lowest energy orientations, the two radicals have parallel $z$ axes.
Figure 2 shows the effect of (restricted) isotropic rotational diffusion $\left(D_{x}=D_{y}=D_{z}=D=(6 \tau)^{-1}\right)$ of the primary radical on the singlet yield profile of this toy radical pair. The recombination lifetime of the radical pair is $10 \mu \mathrm{s}$, which is the minimum time required for the emergence of a noticeable spike. A longer lifetime would allow more time for spin relaxation to occur and consequently the system would relax completely to equilibrium under a wider range of conditions. By keeping the recombination lifetime as short as possible, we are able to study with higher resolution how different physical conditions affect the relaxation rate.

The rates of the various relaxation pathways are proportional to the spectral densities $J(\omega)$ at zero frequency or at the transition frequency between different energy eigenstates of the static Hamiltonian. Previous theoretical studies of the radical pair mechanism have found that relaxation is fastest when the rotational correlation time $\tau \approx 10 \mathrm{~ns}$, such that $h / \tau$ approximately matches the energy scale of the static Hamiltonian. ${ }^{38}$ For longer correlation times, relaxation is slower, but the compass precision is compromised by the disorder associated with molecular motions that are effectively static during the lifetime of the radicals. ${ }^{43}$ Consequently, we only treat correlation times of $10 \mathrm{~ns}$ or less. The types of motion we particularly wish to consider, i.e., restricted rotations of small sections of the magnetoreceptor, are likely to fall within this higher frequency range. For example, at room temperature in aqueous solution, flavin 


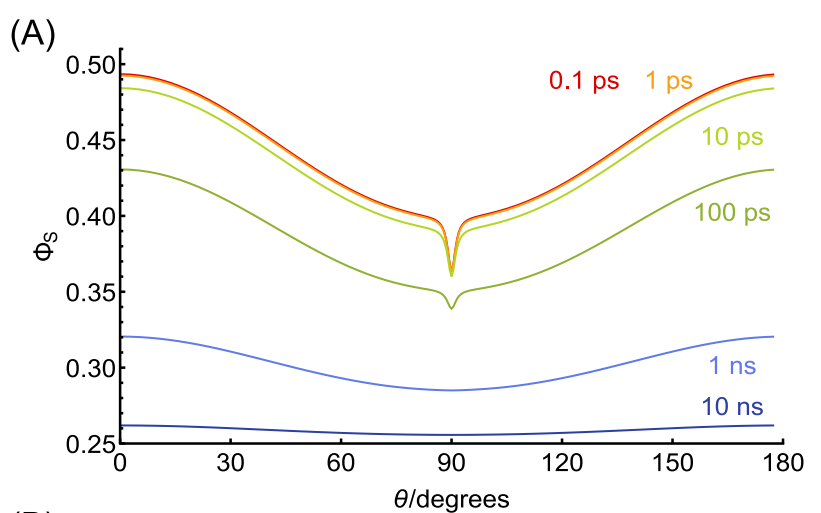

(B)

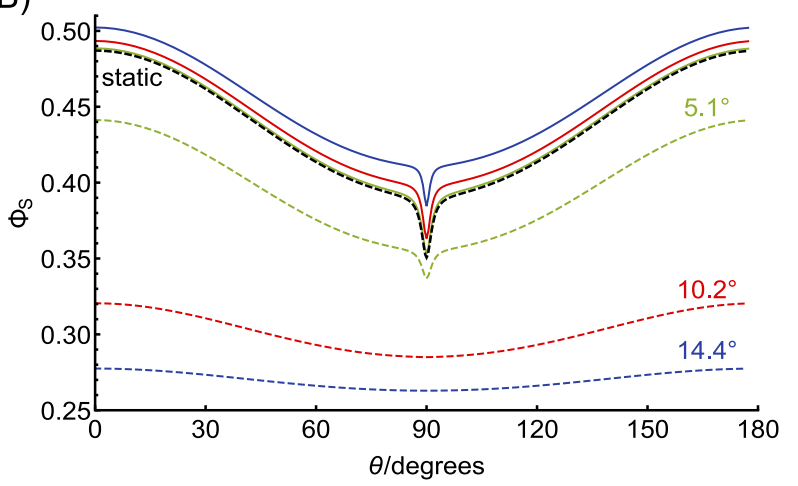

FIG. 2. Relaxation of a four-spin system. The primary radical contains a spin-1 nucleus with hyperfine components $\left[A_{x x}, A_{y y}, A_{z z}\right]$ $=[-0.2,-0.2,1.7569] \mathrm{mT}$. The secondary radical also contains a spin-1 nucleus, with hyperfine components $[0.0,0.0,1.0812] \mathrm{mT}$. The $z$ axes of the two hyperfine tensors are parallel. The radical pair recombination lifetime is $10 \mu \mathrm{s}$. The external field strength is $50 \mu \mathrm{T}$. (a) The primary radical undergoes isotropic rotational diffusion within a potential that allows tumbling of the $z$ axis with a mean deviation of $10^{\circ}(A=100)$. The rotational correlation time increases from $0.1 \mathrm{ps}$ to $10 \mathrm{~ns}$, as indicated. (b) The primary radical undergoes isotropic rotational diffusion within a series of potentials, whose mean deviation is indicated on the graph. Solid lines: $\tau=0.1 \mathrm{ps}$, where the hyperfine couplings are effectively averaged and there is very little relaxation. Dashed lines: $\tau=1 \mathrm{~ns}$, at which strong relaxation occurs. The singlet yield profile corresponding to an ensemble of static, aligned radical pairs is shown by the dashed, black line.

mononucleotide tumbles with a correlation time of around 155 ps. ${ }^{57,58}$

Figure 2(a) shows that relaxation, which is almost complete $\left(\Phi_{\mathrm{S}} \approx 0.25\right)$ when $\tau=10 \mathrm{~ns}$, becomes slower as $\tau$ is decreased to $0.1 \mathrm{ps}$. The amplitude of the broad background curve gradually recovers and the spike becomes visible. For correlation times as short as $1 \mathrm{ps}$, there is negligible relaxation.

Inspection of Eq. (21) and the calculated spectral densities reveals that shorter correlation times (corresponding to larger values of $\varepsilon$ ) result in decreased spectral density at all relevant frequencies. The observed slower relaxation rate is therefore a consequence of both smaller $J(0)$ and $J(\omega)$.

Relaxation rates are also proportional to the mean squared amplitude of the fluctuating magnetic interactions that drive the relaxation. In this case, the relevant fluctuations are in the strength and direction of the field experienced by the electron spin in the moving radical due to the magnetic nuclei to which it is coupled. In less steep potentials, the greater range of orientations sampled by the moving radical leads to larger amplitude fluctuations in the components of the hyperfine tensor in the laboratory frame. Consequently, we observe faster spin relaxation. This is apparent in the increasing gap between the $\tau=0.1 \mathrm{ps}$ (solid lines) and $\tau=1 \mathrm{~ns}$ (dashed lines) traces in Fig. 2(b) as $A$ in Eq. (15) is decreased.

Despite the absence of significant relaxation, the static form of the singlet yield profile is not necessarily recovered in the limit of very fast motion. In the fast motion regime, different orientations of the radical become indistinguishable on the time scale of the coherent spin evolution. As a result, the singlet yield profile reflects the influence of an effective hyperfine interaction, equivalent to the weighted average of the hyperfine tensor over all the orientations of the moving radical. As the value of $A$ (Eq. (15)) is decreased, a greater range of orientations is sampled by the moving radical, enhancing the difference between the static and motionally averaged hyperfine tensors. This is illustrated in Fig. 2(b) by the difference in the singlet yield profile at $\tau=0.1 \mathrm{ps}$ (solid lines) across the three potentials shown.

\section{B. Optimal hyperfine interactions}

The capacity for fast molecular motion to alter the form of the effective hyperfine interaction naturally raises the question of whether motion could improve the ability of a radical pair to function as a compass.

If we assume, in line with previous studies, ${ }^{27,38}$ that optimum compass function is achieved by maximising the singlet yield anisotropy,

$$
\Delta \Phi_{\mathrm{S}}=\left|\Phi_{\mathrm{S}}(\theta=0)-\Phi_{\mathrm{S}}(\theta=\pi / 2)\right|,
$$

it is most favourable for the hyperfine tensor to have the form $\left[0,0, A_{z z}\right]$, i.e., $T_{x x}=T_{y y}=-T_{z z} / 2=-a_{\text {iso }}$. For a hyperfine tensor that instead has $\left|T_{x x}\right|>\left|a_{\text {iso }}\right|$, averaging away some of the anisotropic component via molecular rotation would therefore improve the compass signal. Since some degree of motion is unavoidable in real systems, it could be better to have an ostensibly "non-optimal" static hyperfine interaction that would be improved by motional averaging than an "optimal" one that would be degraded.

The potential advantage offered by rotational averaging is offset by the degree of relaxation caused by the motion. This is illustrated in Fig. 3 using the example of a radical pair containing a single spin-1 nucleus. The electron in one radical is coupled to the nuclear spin with one of two different hyperfine tensors. This radical undergoes isotropic rotational diffusion in an ordering potential with an average deviation of $27^{\circ}(A=15)$. One hyperfine tensor has been designed to be ideal for a static, aligned system (orange lines). The other has a larger anisotropic component that will produce an ideal effective hyperfine tensor when averaged over the potential (blue lines).

In the limit of very fast rotational diffusion (short correlation times), the highest singlet yield anisotropy is produced by the more anisotropic hyperfine tensor (blue lines), which is favourably averaged by the motion. However, the larger anisotropy also induces faster relaxation, ${ }^{38}$ which increasingly degrades the singlet yield anisotropy as the correlation time gets longer. The point at which the less anisotropic hyperfine tensor (orange lines) becomes more 

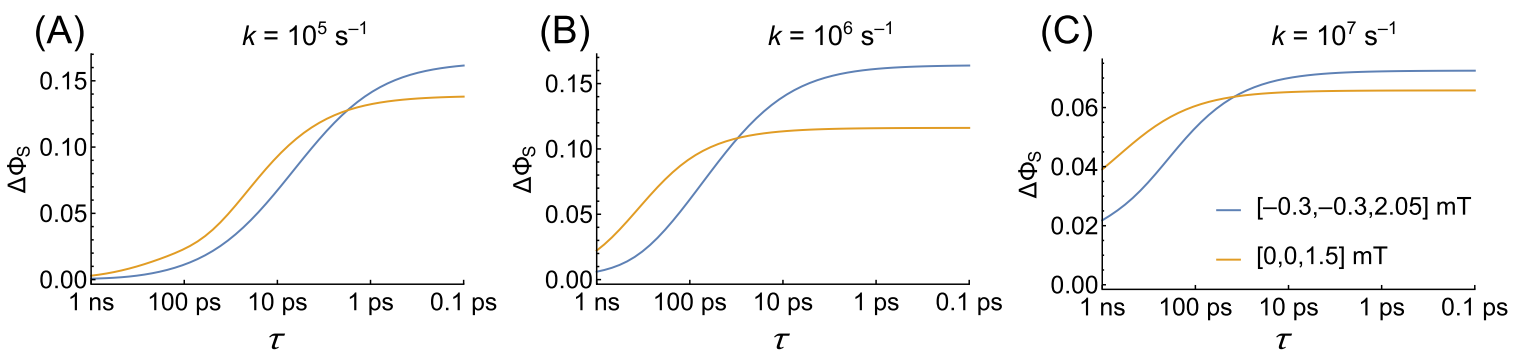

FIG. 3. Example of constructive rotational averaging for a radical pair containing a single spin-1 nucleus. The radical undergoes isotropic diffusion in a potential with a mean deviation of $27^{\circ}$. The hyperfine coupling for this nucleus is either ideally designed to maximise the singlet yield anisotropy in the absence of motion (orange lines), or is averaged to ideal by the molecular rotation (blue lines). The isotropic component $a_{0}^{(0)}=0.5 \mathrm{mT}$. The external field strength is $50 \mu \mathrm{T}$ and the field is applied in the $y z$ plane of the primary radical such that $\varphi=\pi / 2$. (a) $k=10^{5} \mathrm{~s}^{-1}$. (b) $k=10^{6} \mathrm{~s}^{-1}$. (c) $k=10^{7} \mathrm{~s}^{-1}$.

favourable is strongly dependent on the rate constant for radical pair recombination, which dictates how much time is available for relaxation to occur.

When a spike in the singlet yield is taken to be an essential feature of the compass, the picture changes completely. The sensitivity of the compass is measured by the length of the spike, rather than by the singlet yield anisotropy. In addition, the spike should have a narrow width to maximise the compass precision.

The width and length of the spike can be measured by fitting the singlet yield profile to the following function, which models the smoothly varying background as a sinusoidal curve and the spike as a Lorentzian function with amplitude $H$ and width $2 w$ at half its maximum height,

$$
F(\theta)=a+b \cos ^{2} \theta-\frac{H w^{2}}{\left(\theta-\theta_{0}\right)^{2}+w^{2}} .
$$

Fig. 4 shows how the length and width of the spike vary with the size of the components of the hyperfine tensor for a radical pair containing a single spin-1 nucleus in each radical. The hyperfine tensors in the primary and secondary radical are $\left[A_{x x}, A_{x x}, A_{z z}\right]$ and $[0,0,1.08] \mathrm{mT}$, respectively. Two features are immediately striking. First, the values of both parameters increase in the same direction, making it impossible to simultaneously optimise the compass precision and sensitivity. Second, the dimensions of the spike depend only on the ratio $A_{z z} / A_{x x}$, rather than the absolute values of these components. The relative signs of the components were also found to have no impact on the shape of the spike. For a spike with full width $2 w \leq 5^{\circ}$ the principal components of the hyperfine tensor in the primary radical needed to satisfy $A_{z z} / A_{x x} \geq 6.5$. This inequality seems to be approximately valid when more nuclei are included in the spin system, but this has not been explored in detail.

\section{Flavin-tryptophan radical pair}

Much of the relaxation behaviour observed in small, model spin systems can be readily generalised to larger radicals, although with a greater number of interactions it becomes harder to predict the exact form of the interactions that will optimise the compass.

Here, we consider a system with 8 nuclear spins (see Figs. 5(a) and 5(b)), based on the flavin-tryptophan radical pair in cryptochrome. The primary radical contains the three largest hyperfine couplings from $\mathrm{FAD}^{\bullet-}(\mathrm{N} 5, \mathrm{~N} 10, \mathrm{H} 6),{ }^{27}$ whilst the secondary radical consists of the $\mathrm{N} 1, \mathrm{H} 1, \mathrm{H} 4$, $\mathrm{H} 7$, and $\mathrm{H} \beta$ nuclei of $\mathrm{TrpH}^{\bullet+}$ (supplementary material $\mathrm{S} 4^{51}$ ).

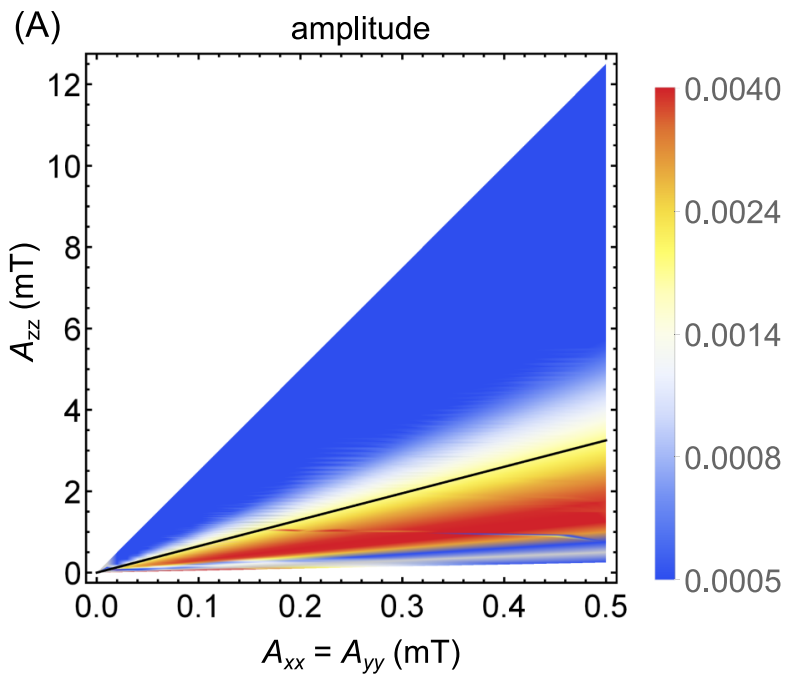

(B) width / degrees

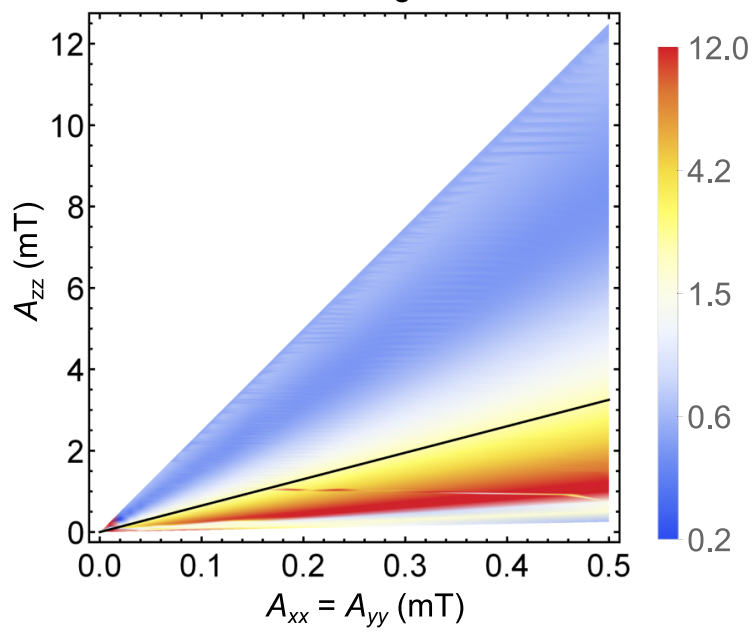

FIG. 4. Spike amplitude (a) and width (b) as a function of the principal components of the hyperfine tensor in the primary radical. Each radical in the pair contains a single spin-1 nucleus. The primary radical has hyperfine components $\left[A_{x x}, A_{y y}, A_{z z}\right]$. The secondary radical has hyperfine components $[0,0,1.0812] \mathrm{mT} . k=10^{5} \mathrm{~s}^{-1}$. The external field strength is $50 \mu \mathrm{T}$ and the field is applied in the $y z$ plane of the primary radical such that $\varphi=\pi / 2$. Both radicals are static. The solid black lines indicate a spike width of $2 w=5^{\circ}$. 


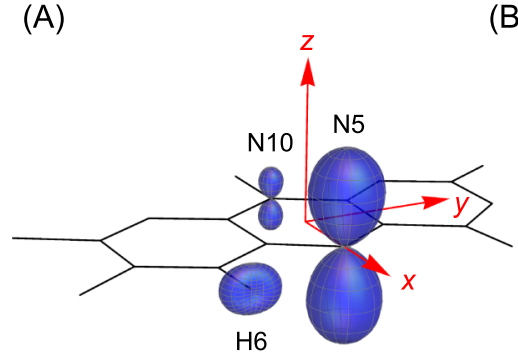

(B)

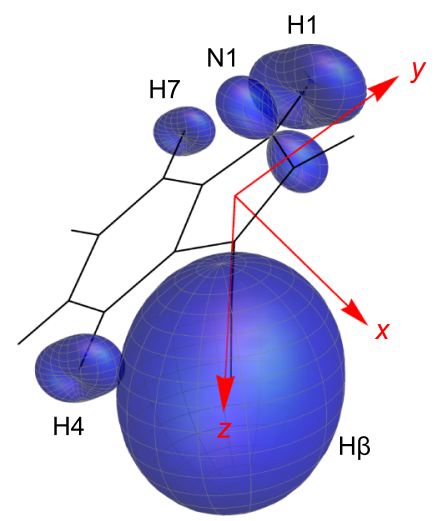

(C)

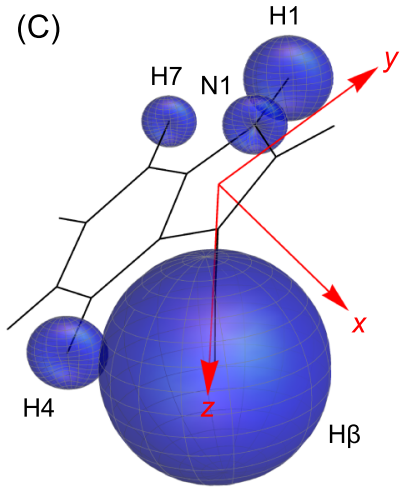

FIG. 5. Representations of the hyperfine interactions included in (a) the flavin radical FAD ${ }^{\bullet-}$ and (b) the tryptophan radical TrpH ${ }^{\bullet+}$. The corresponding hyperfine tensors are given in the supplementary material S4. ${ }^{51}$ The relative orientation of the two radicals is taken to be that of FAD and Trp-342 (the terminal tryptophan of the Trp-triad) in the crystal structure of Drosophila melanogaster cryptochrome. ${ }^{65,66}$ (See supplementary material S5. ${ }^{51}$ ) (c) Hyperfine interactions in the tryptophan radical after rotational averaging within a potential with $A=100$ and the symmetry axis ( $z$ axis) defined by the C3-C $\beta$ bond.

These nuclei have previously been identified as dominating both the static singlet yield profile of the flavin-tryptophan radical pair ${ }^{27}$ and many of its relaxation pathways. ${ }^{39}$ In the flavin radical the principal $(z)$ axis is defined as the axis perpendicular to the plane of the aromatic ring system. In the tryptophan radical, the $z$ axis is defined by the $\mathrm{C} 3-\mathrm{C} \beta$ bond, which joins the indole group to the rest of the amino acid sidechain. In all calculations the $z$ axis of the ordering potential is aligned with the $z$ axis of the moving radical before motion is included.

The singlet yield is calculated as a function of the direction $\theta$ of the Earth's magnetic field, which is applied in the $y z$ plane of the primary radical $(\varphi=\pi / 2)$. This crosssection was chosen because of the clear separation of the small spike at $\theta \approx 30^{\circ}-40^{\circ}$, attributed to $\mathrm{N} 1$ in the tryptophan radical, and the larger spike at $\theta=90^{\circ}$, created by the N5 and $\mathrm{N} 10$ hyperfine interactions in the flavin radical. The spike at $\theta \approx 30^{\circ}-40^{\circ}$ is sensitive to the number of nuclei included in the simulation and consequently we focus on the effect of motion on the spike at $\theta=90^{\circ}$. A full 3D representation of the singlet yield for a static flavin-tryptophan radical pair can be found in Ref. 43.

Fig. 6(a) shows the impact of constrained isotropic rotational diffusion of the model flavin radical on the singlet yield anisotropy (Eq. (22)). The recombination lifetime is $1 \mu \mathrm{s}$, which is too short to allow spikes to develop. In the limit of very fast motion, the system is effectively in a regime of static order, with a set of motionally averaged hyperfine tensors. For $A=100$ and $A=400$, the motionally averaged interactions yield a marginally stronger compass signal, whilst averaging over larger amplitude motions degrades the signal. As for small spin systems, the relaxation rate increases with the amplitude of the wobbling such that the optimal size of motion depends on the diffusion rate.

For longer-lived radical pairs it is observed that motional averaging of the flavin hyperfine tensors, even within highly restricted potentials, reduces the length of the spike (see Figs. 6(b) and 6(c)). The spike, as well as the overall anisotropy, is further attenuated by relaxation as the correlation time is increased from $\tau=0.1 \mathrm{ps}$ to $\tau=1 \mathrm{~ns}$, although the width of the spike is unchanged. In the $A=400$ potential shown in Fig. 6(b), the spike is barely visible for $\tau=100 \mathrm{ps}$ and has completely disappeared for $\tau=1 \mathrm{~ns}$. As $A$ is reduced, the rate of relaxation increases and the spike disappears more rapidly. The uniaxial nature of the N5 and N10 hyperfine interactions in the flavin radical means that the observed relaxation is primarily caused by the wobbling motion of the molecular $z$ axis, rather than diffusion around the $z$ axis itself. To demonstrate this, Fig. 6(b) includes a trace corresponding to $D_{z}=1.67 \times 10^{8} \mathrm{~s}^{-1}$ and $D_{x}=D_{y}=1.67 \times 10^{12} \mathrm{~s}^{-1}$ (i.e., $\left.\eta=10^{-4}\right)$. Although substantial relaxation occurs when all three diffusion rate constants equal $1.67 \times 10^{8} \mathrm{~s}^{-1}$, the signal is only slightly degraded compared to the limit of fast isotropic diffusion when only $D_{z}$ has this value.

The hyperfine interactions in a static tryptophan radical do not have the same mutually parallel uniaxial symmetry as do N5 and N10 in the flavin radical. Consequently, diffusion of tryptophan around the $\mathrm{C} 3-\mathrm{C} \beta$ bond is associated with large fluctuations in the electron-nuclear interactions, driving fast relaxation. Motional averaging around this bond also greatly reduces the anisotropy of the hyperfine interactions (Fig. 5(c)). The resulting singlet yield profile has a more strongly anisotropic background but the spike is barely visible, even in a regime of very slow relaxation (Fig. 6(c)).

By contrast, averaging the hyperfine interactions around an axis perpendicular to the plane of the indole ring produces a set of hyperfine tensors with a uniaxial symmetry that is missing from their static counterparts, whilst still preserving the anisotropy of the N1 hyperfine tensor. The increased symmetry of the interactions greatly enhances the spike (see supplementary material $\mathrm{S}^{51}$ ).

In a protein environment, the motion of the radicals is likely to be constrained around all three axes and hence might be better described by diffusion within a biaxial potential. Biaxial potentials require a more complex treatment than uniaxial potentials and are not within the scope of the method presented here. However, to give a sense of how biaxially constrained motion might affect the compass, Fig. 6(d) shows the singlet yield profile for a static flavin-tryptophan radical 
(A)

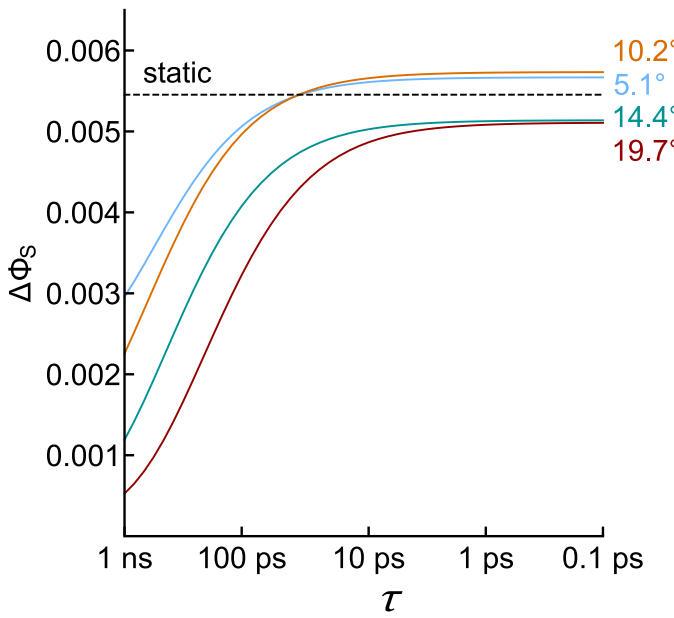

(C)

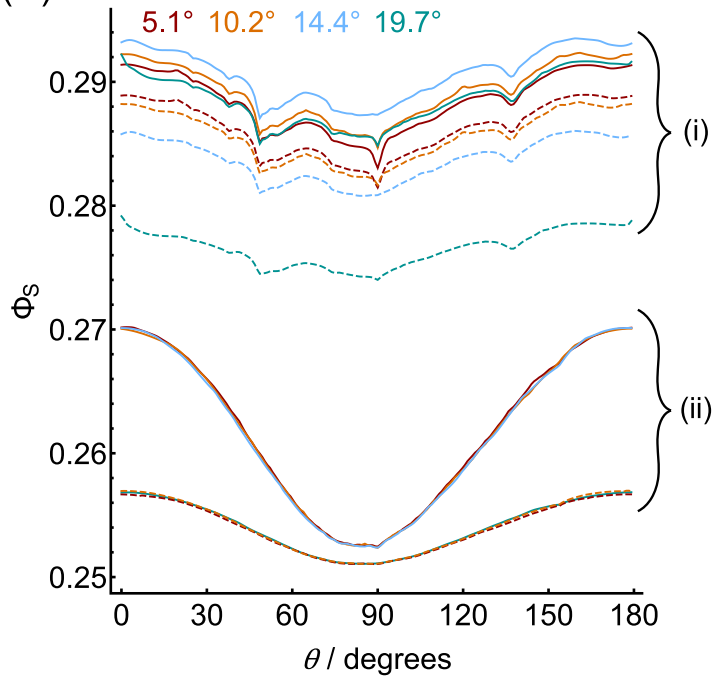

(B)

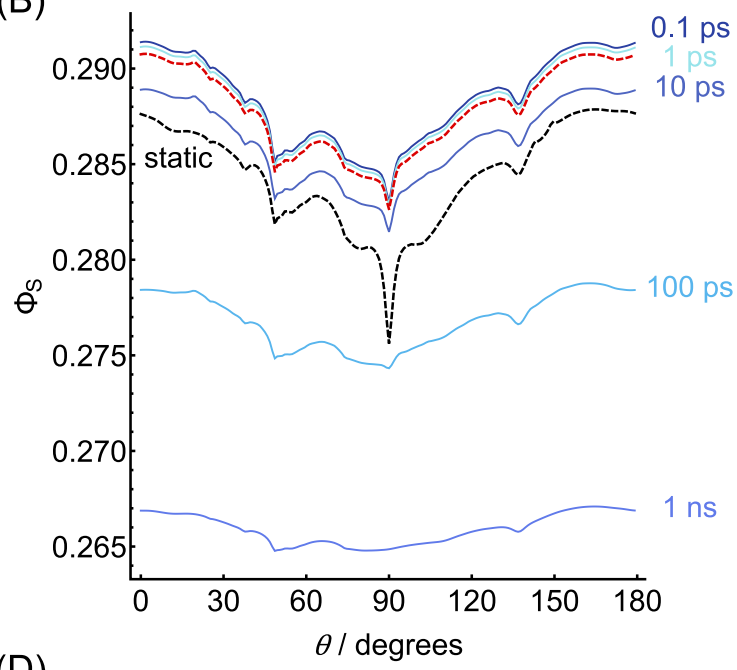

(D)

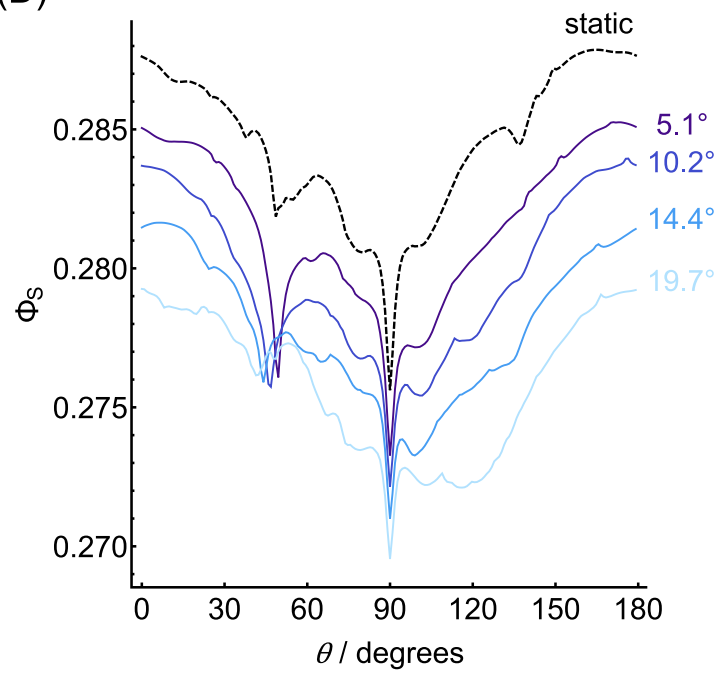

FIG. 6. Relaxation of a ten-spin system. The primary radical contains the N5, N10, and H6 nuclei from FAD ${ }^{\bullet-}$. The secondary radical consists of N1, H1, H4, $\mathrm{H} 7$, and $\mathrm{H} \beta$ from $\operatorname{TrpH}^{\bullet+}$. The external field strength is $50 \mu \mathrm{T}$ and the field is applied in the $y z$ plane of the primary radical such that $\varphi=\pi / 2$. (a) Isotropic rotational diffusion of the primary radical in a series of potentials of different mean deviation (see Table I). The radical pair recombination lifetime is $1 \mu \mathrm{s}$, so no spikes are observable. Only the singlet yield anisotropy, Eq. (22), is reported. The dashed, black line indicates the singlet yield anisotropy produced by a static, aligned ensemble of radical pairs. (b) Isotropic rotational diffusion of the primary radical within a potential that allows tumbling of the $z$ axis with a mean deviation of $5^{\circ}$. The rotational correlation time increases from $0.1 \mathrm{ps}$ to $1 \mathrm{~ns}$, as indicated. The black, dashed line indicates the reaction yield for a static, aligned ensemble of radical pairs. The red line shows the reaction yield when the diffusion is anisotropic, with $D_{x}=D_{y}=1.67 \times 10^{12} \mathrm{~s}^{-1}$ and $D_{z}=1.67 \times 10^{8} \mathrm{~s}^{-1}$. The radical pair recombination lifetime is $10 \mu \mathrm{s}$. (c) Isotropic rotational diffusion of (i) the primary radical and (ii) the secondary radical in a series of potentials of different mean deviation, as indicated. Solid lines indicate a rotational correlation time of $0.1 \mathrm{ps}$, for which there is no significant relaxation. Dashed lines indicate a rotational correlation time of $10 \mathrm{ps}$. The radical pair recombination lifetime is $10 \mu \mathrm{s}$. (d) Singlet yield profiles for an ensemble of static, aligned radical pairs. The hyperfine tensors for the secondary radical have been numerically averaged over a series of biaxial potentials (Eq. (24)). The mean deviation of the $z$ axis in each potential is indicated on the graph. The corresponding values of $A$ can be found in Table I. The black dashed line indicates the reaction yield for the original, unaveraged system. The radical pair recombination lifetime is $10 \mu \mathrm{s}$.

pair for which the hyperfine interactions in the tryptophan radical have been numerically averaged over a potential with the form

$$
\begin{aligned}
U(\alpha, \beta, \gamma) & =A \cos ^{2}\left(\frac{\beta}{2}\right) \cos (\alpha+\gamma) \\
& =\frac{A}{2}\left(\mathfrak{D}_{1,1}^{(1)}(\alpha, \beta, \gamma)+\mathfrak{D}_{-1,-1}^{(1)}(\alpha, \beta, \gamma)\right) .
\end{aligned}
$$

Although the anisotropy of the hyperfine interactions is reduced as $A$ is decreased (as evidenced by the reduction in the sharp feature at $\sim 40^{\circ}$, produced by the $\mathrm{N} 1$ interaction), the length of the spike at $\theta=90^{\circ}$ is not significantly altered.

\section{DISCUSSION}

\section{A. Flavin radical}

In the flavin-tryptophan radical pair, it is the flavin radical that is primarily responsible for producing the sharp spike observed at $\theta=90^{\circ}$, when the recombination lifetime is $\geq 10 \mu \mathrm{s}$. To maximize the sensitivity of the compass, the spike should be as large as possible, whilst still remaining narrow enough to provide the required compass precision. As the length and width of the spike increase simultaneously in response to changes in the hyperfine tensors, this implies that the "best" spikes have the maximum tolerable width, in order to optimise the sensitivity. Experiments have shown that 
birds can detect the axis of the Earth's magnetic field with a precision between $2^{\circ}$ and $5^{\circ} .44,45$ We can use this as an estimate for the width $2 w$ of an ideal spike. In a static system, with a recombination lifetime of $10 \mu \mathrm{s}$, the flavin radical produces a spike roughly $5^{\circ}$ wide, making it very well-suited to its role as a component of a sensitive, precise compass. Previous studies have also found that flavin radicals are almost ideally designed to produce a large singlet yield anisotropy when radical pair recombination is faster and spikes are not observable. $^{27}$

We therefore conclude that there is little scope for improving the hyperfine tensors in flavin by rotational averaging. This is supported by the simulations presented here, which indicate that even highly restricted molecular tumbling degrades the length of the spike and the overall anisotropy of the singlet yield. For optimal compass function, motion of the flavin radical should be constrained as much as possible to minimize the detrimental effects of spin relaxation.

Fortunately, the location of FAD, buried inside cryptochrome, means that it is well-placed to have its motion constrained by the surrounding sections of the protein. Molecular dynamics simulations of cryptochrome 1 from Arabidopsis thaliana suggest that librations of the flavin radical are restricted to approximately $5^{\circ},{ }^{39}$ which we have shown to be compatible with a functioning compass when the rotational correlation time is $100 \mathrm{ps}$ or less and the recombination lifetime is $10 \mu \mathrm{s}$. For longer-lived radical pairs, relaxation processes have more time to take effect and are consequently more damaging. To maintain a functioning compass with longer-lived radicals, the correlation time for flavin wobbling would need to be shorter. Alternatively, the loss of some anisotropy or "spikiness" could be compensated for by amplification further down the signalling pathway. ${ }^{33}$

\section{B. Tryptophan radical}

Tryptophan has a greater number of large, anisotropic hyperfine interactions than flavin. Furthermore, many of the interactions are rhombic rather than axial, with large components along more than one axis. These differences mean that very different sorts of motion are favourable for tryptophan compared to flavin.

One consequence of the rhombicity of the hyperfine interactions in the tryptophan radical is that free diffusion around the $\mathrm{C} 3-\mathrm{C} \beta$ bond dominates both the form of the motionally averaged hyperfine tensors and the rate of relaxation. Although this type of motion has been observed for certain exposed tryptophan residues, ${ }^{59,60}$ it is unlikely to occur in a crowded protein environment. However, the calculations presented here do still provide some pertinent information about the effect of tryptophan wobbling on the properties of the compass.

Lying at the surface of the protein, the tryptophan radical in cryptochrome has been shown by molecular dynamics simulations to undergo librations of the indole ring of up to $10^{\circ} .{ }^{39}$ This is less restricted motion than would ideally be allowed for the flavin radical. However, from both Figs. 6(c) and 6(d), it is clear that the amplitude of tryptophan wobbling does not noticeably affect the dimensions of the spike, provided it is constrained enough not to average away all the anisotropy of the hyperfine interactions. This is consistent with the observation that the spike is primarily shaped by the hyperfine interactions in the flavin radical. If the motion was sufficiently fast, $10^{\circ}$ librations of the indole ring would probably not destroy the compass function.

Although in the fast motion regime, constrained tryptophan wobbling is unlikely to have much effect on the shape of the compass signal, the large number of highly anisotropic hyperfine interactions in the tryptophan radical means that even small librations are accompanied by large fluctuations in the hyperfine tensors. This is especially true for rotational diffusion around the $\mathrm{C} 3-\mathrm{C} \beta$ bond. Large fluctuations in the interactions drive fast relaxation, meaning that particularly fast librations of the tryptophan radical are likely to be required for a functioning compass. Additionally, in a real system, both radicals will wobble around and relax simultaneously, leading to a faster loss of spin coherence than if (as here) one radical is treated as static. A full investigation of the required motional constraints demands a more complex treatment than that presented here. However, some insight into the effect of correlated relaxation of the two radicals is given in Ref. 61.

\section{Alternative secondary radicals}

It has been suggested on several occasions that there may be other, more suitable, candidates than tryptophan for the secondary radical. ${ }^{27,62-64}$ The present study suggests some criteria that an improved secondary radical might meet. First, having fewer and smaller hyperfine interactions in the secondary radical would result in slower relaxation because of the weaker fluctuating fields experienced by the electron spin. Furthermore, previous studies of static systems have shown that a larger singlet yield anisotropy is obtained when the secondary radical has fewer and smaller hyperfine interactions. $^{27}$

Second, a better secondary radical might have more axially symmetric, mutually aligned hyperfine tensors. The improvement in the singlet yield profile that arises from motional averaging of the tryptophan tensors around the $z$ axis suggests that increased symmetry enhances the compass sensitivity. In addition, diffusion could then occur around the symmetry axis without causing significant relaxation, whatever the diffusion rate. This would mean that fewer constraints would need to be placed on the molecular motion to preserve the compass signal.

Finally, as the dimensions of the spike are largely determined by the hyperfine tensors in the primary radical, we have seen that the secondary radical could be allowed to undergo less constrained motion, without destroying the compass signal. This implies that the secondary radical might not necessarily need to be tightly bound to the protein, particularly if its hyperfine interactions met the criteria described above to minimise relaxation. 


\section{CONCLUSIONS}

In this study, we have explored the impact of rotational diffusion of small sections of the magnetoreceptor protein on the sensitivity and precision of the compass signal. We have demonstrated that molecular motion can greatly alter the criteria for optimal compass design. The definition of an ideal hyperfine interaction depends strongly on the lifetime of the radical pair, the amplitude and frequency of its wobbling motion, and the desired shape of the singlet yield profile. Relaxation is slowest when molecular librations are small-scale, with short correlation times. However, rotational averaging of the hyperfine tensors by less constrained motions can be beneficial in a regime of slow relaxation. In compass design, a balance must always be struck between optimising important features of the singlet yield profile in the static limit and minimising the amount of relaxation induced by molecular motion.

We have studied in detail the effect of molecular motion on the proposed flavin-tryptophan radical pair magnetoreceptor. We conclude that, in its static form, the flavin radical is wellsuited for a radical pair compass. Rotational averaging of the hyperfine tensors therefore offers no advantage and librational motion of the flavin ring should be as constrained as possible to minimise relaxation. Within the $5^{\circ}$ ordering potential predicted by molecular dynamics simulations, the compass signal is preserved over a range of physically realisable diffusion rates.

By contrast, rotational diffusion of tryptophan causes fast relaxation, unless the correlation time is short. However, the dimensions of the spike are relatively insensitive to small changes in the hyperfine tensors caused by librational motion of the indole ring. The tryptophan radical could therefore be allowed less restricted motion than the flavin radical, in keeping with its position close to the surface of cryptochrome. Secondary radicals with fewer, smaller, and more symmetric hyperfine tensors could provide a better alternative to tryptophan in forming a radical pair compass with a flavin radical.

\section{ACKNOWLEDGMENTS}

This work was supported by the European Research Council (ERC; under the European Union's 7th Framework Programme, FP7/2007-2013/ERC Grant No. 340451) and the U.S. Air Force (USAF) Office of Scientific Research (Air Force Materiel Command, USAF Award No. FA9550-14-10095).

${ }^{1}$ W. Wiltschko and R. Wiltschko, Magnetic Orientation in Animals (Springer Verlag, 1995)

${ }^{2}$ P. Berthold, Bird Migration. A General Survey (Oxford University Press, 2001).

${ }^{3}$ H. Mouritsen, D. Heyers, and O. Güntürkün, Annu. Rev. Physiol. 78, 133 (2016).

${ }^{4}$ W. Wiltschko and R. Wiltschko, Science 176, 62 (1972).

${ }^{5}$ H. Mouritsen and P. J. Hore, Curr. Opin. Neurobiol. 22, 343 (2012).

${ }^{6}$ K. Maeda, K. B. Henbest, F. Cintolesi, I. Kuprov, C. T. Rodgers, P. A. Liddell, D. Gust, C. R. Timmel, and P. J. Hore, Nature 453, 387 (2008).

${ }^{7}$ K. B. Henbest, P. Kukura, C. T. Rodgers, P. J. Hore, and C. R. Timmel, J. Am. Chem. Soc. 126, 8102 (2004).

${ }^{8}$ T. Ritz, P. Thalau, J. B. Phillips, R. Wiltschko, and W. Wiltschko, Nature 429, 177 (2004).

${ }^{9}$ H. Mouritsen and T. Ritz, Curr. Opin. Neurobiol. 15, 406 (2005).
${ }^{10}$ R. Wiltschko and W. Wiltschko, BioEssays 28, 157 (2006).

${ }^{11}$ K. Schulten, C. E. Swenberg, and A. Weller, Z. Phys. Chem. 111, 1 (1978).

${ }^{12}$ P. J. Hore and H. Mouritsen, Annu. Rev. Biophys. 45, 299 (2016).

${ }^{13}$ W. Wiltschko and R. Wiltschko, J. Exp. Biol. 204, 3295 (2001).

${ }^{14}$ R. Wiltschko, K. Stapput, P. Thalau, and W. Wiltschko, J. R. Soc., Interface 7, S163 (2010).

${ }^{15}$ S. Engels, N.-L. Schneider, N. Lefeldt, C. M. Hein, M. Zapka, A. Michalik, D. Elbers, A. Kittel, P. J. Hore, and H. Mouritsen, Nature 509, 353 (2014).

${ }^{16} \mathrm{P}$. Thalau, T. Ritz, K. Stapput, R. Wiltschko, and W. Wiltschko, Naturwissenschaften 92, 86 (2005).

${ }^{17}$ T. Ritz, S. Adem, and K. Schulten, Biophys. J. 78, 707 (2000).

${ }^{18}$ T. Biskup, E. Schleicher, A. Okafuji, G. Link, K. Hitomi, E. D. Getzoff, and S. Weber, Angew. Chem., Int. Ed. 48, 404 (2009).

${ }^{19}$ M. Liedvogel and H. Mouritsen, J. R. Soc., Interface 7, S147 (2010).

${ }^{20}$ C. Niessner, S. Denzau, J. C. Gross, L. Peichl, H. J. Bischof, G. Fleissner, W. Wiltschko, and R. Wiltschko, PLoS One 6, 1 (2011).

${ }^{21}$ H. Mouritsen, U. Janssen-Bienhold, M. Liedvogel, G. Feenders, J. Stalleicken, P. Dirks, and R. Weiler, Proc. Natl. Acad. Sci. U. S. A. 101, 14294 (2004).

${ }^{22}$ A. Möller, S. Sagasser, W. Wiltschko, and B. Schierwater, Naturwissenschaften 91, 585 (2004).

${ }^{23}$ Most bird species that have been shown to have a light-dependent magnetic compass are nocturnal migrants. Just how much light is required for the compass to operate is discussed in Ref. 12.

${ }^{24}$ K. Maeda, A. J. Robinson, K. B. Henbest, H. J. Hogben, T. Biskup, M. Ahmad, E. Schleicher, S. Weber, C. R. Timmel, and P. J. Hore, Proc. Natl. Acad. Sci. U. S. A. 109, 4774 (2012).

${ }^{25}$ S. Weber, T. Biskup, A. Okafuji, A. R. Marino, T. Berthold, G. Link, K. Hitomi, E. D. Getzoff, E. Schleicher, and J. R. Norris, J. Phys. Chem. B 114, 14745 (2010)

${ }^{26}$ K. B. Henbest, K. Maeda, P. J. Hore, M. Joshi, A. Bacher, R. Bittl, S. Weber, C. R. Timmel, and E. Schleicher, Proc. Natl. Acad. Sci. U. S. A. 105, 14395 (2008).

${ }^{27}$ A. A. Lee, J. C. S. Lau, H. J. Hogben, T. Biskup, D. R. Kattnig, and P. J. Hore, J. R. Soc., Interface 11, 20131063 (2014).

${ }^{28}$ F. Cintolesi, T. Ritz, C. W. M. Kay, C. R. Timmel, and P. J. Hore, Chem. Phys. 294, 385 (2003)

${ }^{29}$ O. Efimova and P. J. Hore, Biophys. J. 94, 1565 (2008).

${ }^{30}$ C. T. Rodgers and P. J. Hore, Proc. Natl. Acad. Sci. U. S. A. 106, 353 (2009).

${ }^{31}$ J. L. Kirschvink, M. Winklhofer, and M. M. Walker, J. R. Soc., Interface 7, S179 (2010)

${ }^{32}$ I. A. Solov'yov, H. Mouritsen, and K. Schulten, Biophys. J. 99, 40 (2010).

${ }^{33}$ E. Hill and T. Ritz, J. R. Soc., Interface 7, S265 (2010).

${ }^{34} \mathrm{M}$. Winklhofer, IEEE Trans. Magn. 45, 5259 (2009).

${ }^{35}$ J. C. S. Lau, C. T. Rodgers, and P. J. Hore, J. R. Soc., Interface 9, 3329 (2012).

36 J. Cai and M. B. Plenio, Phys. Rev. Lett. 111, 1 (2013).

${ }^{37}$ E. M. Gauger, E. Rieper, J. J. L. Morton, S. C. Benjamin, and V. Vedral, Phys. Rev. Lett. 106, 040503 (2011).

${ }^{38}$ J. C. S. Lau, N. Wagner-Rundell, C. T. Rodgers, N. J. B. Green, and P. J. Hore, J. R. Soc., Interface 7, S257 (2010).

${ }^{39}$ D. R. Kattnig, I. A. Solov'yov, and P. J. Hore, Phys. Chem. Chem. Phys. 18, 12443 (2016).

${ }^{40}$ A. G. Redfield, IBM J. Res. Dev. 1, 19 (1957).

${ }^{41}$ M. Goldman, J. Magn. Reson. 149, 160 (2001).

${ }^{42}$ N. M. Atherton, Electron Spin Resonance (Ellis Horwood Limited, 1973).

${ }^{43}$ H. G. Hiscock, S. Worster, D. R. Kattnig, C. Steers, Y. Jin, D. E. Manolopoulos, H. Mouritsen, and P. J. Hore, Proc. Natl. Acad. Sci. U. S. A. 113, 4634 (2016).

${ }^{44}$ S. Akesson, J. Morin, R. Muheim, and U. Ottosson, Proc. R. Soc. B 268, 1907 (2001)

${ }^{45}$ N. Lefeldt, D. Dreyer, N.-L. Schneider, F. Steenken, and H. Mouritsen, J. Exp. Biol. 218, 206 (2015).

${ }^{46}$ U. E. Steiner and T. Ulrich, Chem. Rev. 89, 51 (1989).

${ }^{47}$ P. J. Hore, Nuclear Magnetic Resonance, 2nd ed. (Oxford University Press, Oxford, 2015).

${ }^{48}$ C. R. Timmel, U. Till, B. Brocklehurst, K. A. Mclauchlan, and P. J. Hore, Mol. Phys. 95, 71 (1998).

${ }^{49}$ P. L. Nordio and P. Busolin, J. Chem. Phys. 55, 5485 (1971).

${ }^{50}$ I. Kuprov, N. Wagner-Rundell, and P. J. Hore, J. Magn. Reson. 184, 196 (2007).

${ }^{51}$ See supplementary material at http://dx.doi.org/10.1063/1.4958624 for hyperfine data and further details of the Redfield relaxation treatment. 
${ }^{52}$ R. Tarroni and C. Zannoni, J. Chem. Phys. 95, 4550 (1991).

${ }^{53}$ D. A. Varshalovich, A. N. Moskalev, and V. K. Khersonskii, Quantum Theory of Angular Momentum (World Scientific Publishing Co. Pte Ltd., 1988).

${ }^{54}$ E. Berggren, R. Tarroni, and C. Zannoni, J. Chem. Phys. 99, 6180 (1993).

${ }^{55}$ D. E. Manolopoulos and P. J. Hore, J. Chem. Phys. 139, 124106 (2013).

${ }^{56}$ A. M. Lewis, D. E. Manolopoulos, and P. J. Hore, J. Chem. Phys. 141, 044111 (2014).

${ }^{57}$ R. Leenders, M. Kooijman, A. van Hoek, C. Veeger, and A. J. W. G. Visser, Eur. J. Biochem. 211, 37 (1993).

${ }^{58}$ E. W. Evans, J. Li, J. G. Storey, K. Maeda, K. B. Henbest, C. A. Dodson, P. J. Hore, S. R. Mackenzie, and C. R. Timmel, Phys. Chem. Chem. Phys. 17, 18456 (2015).

${ }^{59}$ A. H. A. Clayton and W. H. Sawyer, Biophys. J. 76, 3235 (1999).
${ }^{60}$ S. L. C. Moors, M. Hellings, M. De Maeyer, Y. Engelborghs, and A. Ceulemans, Biophys. J. 91, 816 (2006).

${ }^{61}$ D. R. Kattnig, J. K. Sowa, I. A. Solov'yov, and P. J. Hore, New J. Phys. 18, 063007 (2016).

${ }^{62}$ T. Ritz, R. Wiltschko, P. J. Hore, C. T. Rodgers, K. Stapput, P. Thalau, C. R. Timmel, and W. Wiltschko, Biophys. J. 96, 3451 (2009).

${ }^{63}$ H. J. Hogben, O. Efimova, N. Wagner-Rundell, C. R. Timmel, and P. J. Hore, Chem. Phys. Lett. 480, 118 (2009).

${ }^{64}$ I. A. Solov'yov and K. Schulten, Biophys. J. 96, 4804 (2009).

${ }^{65}$ C. Levy, B. D. Zoltowski, A. R. Jones, A. T. Vaidya, D. Top, J. Widom, M. W. Young, N. S. Scrutton, B. R. Crane, and D. Leys, Nature 495, E3 (2013).

${ }^{66}$ B. D. Zoltowski, A. T. Vaidya, D. Top, J. Widom, M. W. Young, and B. R. Crane, Nature 480, 396 (2011). 